\title{
Evolutionary scenario for W UMa-type stars
}

\author{
K. Stẹpien ${ }^{1}$ and K. Gazeas ${ }^{2}$ \\ ${ }^{1}$ Warsaw University Observatory, email: kst@astrouw.edu.pl \\ ${ }^{2}$ Harvard-Smithsonian Center for Astrophysics, email: kgaze@physics.auth.gr
}

\begin{abstract}
An alternative to TRO model of a W UMa-type star is presented in which the binary is past mass exchange with mass ratio reversal. The secondary is hydrogen depleted and both components are in thermal equilibrium. Evolution in contact is driven by orbital angular momentum loss and mass transfer from the secondary to primary component, similarly as it is observed in Algols. Temperature equalization of both components results from an assumed energy transfer by a large scale flow encircling the whole system in the common envelope.
\end{abstract}

Keywords. stars: activity, binaries: close, stars: evolution, stars: late type

W UMa-type stars are believed to originate from close detached binaries which lose angular momentum (AM) via a magnetized wind until a primary overflows its critical Roche lobe and transfers mass to the secondary. Details of the mass transfer (MT) and further evolution of the binary is a matter of debate. Lucy (1976) developed a model with a secondary swollen due to the transfer of a limited amount of mass $\left(\sim 0.1-0.2 M_{\odot}\right)$. A contact binary is formed but it is out of thermal equilibrium and undergoes thermal relaxation oscillations (TRO, see also Eggleton \& Kiseleva-Eggleton 2002). Its further evolution is driven by an increasing luminosity of the evolving primary, which forces the secular MT from the secondary. An alternative model has been developed by Stẹpień $(2004,2006)$ who observed that the time scale of orbital AM loss of a cool close binary with an initial period equal to a couple of days is the same as the evolutionary time scale of its primary. Due to this coincidence, the primary reaches its Roche lobe when it is close to, or just beyond TAMS. The model assumes that MT following the overflow of the Roche lobe by the primary leads to a common envelope phase during which mass ratio reversal takes place, much the same way as in Algols. Depending on the initial parameters and details of evolution in the common envelope phase, the binary emerges from that phase either directly as a contact binary or as a very short-period Algol transformed subsequently into the contact binary after the surplus AM is lost.

Neglecting spin AM of both components, compared to orbital AM, the AM loss rate of a close binary by a magnetized wind is given by

$$
\frac{\mathrm{d} H_{\text {orb }}}{\mathrm{d} t}=-4.9 \times 10^{41}\left(R_{1}^{2} M_{1}+R_{2}^{2} M_{2}\right) / P_{\text {orb }}
$$

Here AM is in cgs units, period in days, masses and radii in solar units and time in years. The formula is based on semi-empirically determined AML rate of single, cool stars. The supersaturation effect is allowed for by assuming $P_{\text {orb }} \equiv 0.4$ days for periods shorter than 0.4 days.

Together with AM mass is also carried away by magnetized winds. Mass loss rate is often treated as a free parameter, particularly in close binary studies (Eggleton \& Kiseleva-Eggleton 2002 ). We assume, however, that the mass loss rate of each component is the same as of a single star with the same basic characteristics. Mass loss rates of single active stars were recently determined from empirical data by Wood et al. (2002). According to their results, active stars lose mass at a constant rate per surface area, so 

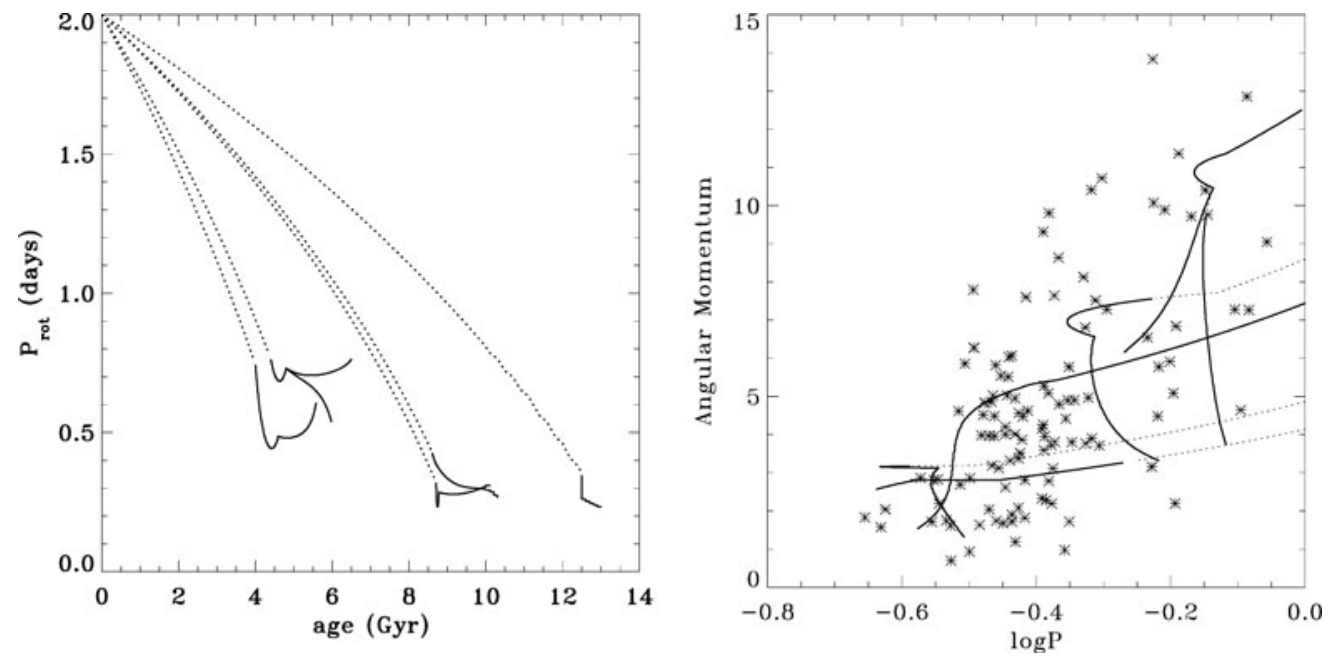

Figure 1. Computed time variations of orbital period of binaries with masses (from left to right): $1.3+1.1,1.3+1.0,1.0+0.8,1.0+0.5$ and $0.9+0.45 M_{\odot}$ (left) and AM variations of the same binaries compared to the observed values of over $100 \mathrm{~W}$ UMa-type stars. Dotted lines denote detached stage and solid lines the contact phase.

that a parametric formula can be used for each component: $\dot{M}_{1,2}=-10^{-11} R_{1,2}^{2}$, where mass loss rates are in $M_{\odot} /$ year, radii in solar units and numbers denote the respective components.

Once a contact binary is formed, energy transfer from the primary to secondary takes place in a way described e.g. by Kähler (2004). He argued that a large scale flow takes place between the inner and outer Roche lobe. The flow is separated from the convective envelope of the secondary by a radiative layer so that, contrary to what Lucy (1976) assumed, the convective layers of both components do not have identical adiabatic constants.

During evolution in contact a self-regulating mechanism develops in which AM loss makes orbit shrink but the secular MT from the secondary widens it. As a result, MT rate adapts itself to the AM loss rate so that contact between both components is maintained. Model calculations show that the resulting average MT rates are about $3-4 \times 10^{-10} M_{\odot} /$ year and typical lifetimes in contact are about 1-1.5 Gyr. Coalescence of both components ends the contact phase. Detailed evolutionary calculations of several sample binaries were obtained by Gazeas \& Stẹpień (2008). Fig. 1 presents period evolution of those binaries starting from the (assumed) initial value of 2 days (left) and comparison of the predicted AM variations with the observed values (right).

\section{References}

Eggleton, P. P. \& Kiseleva-Eggleton, L. 2002, ApJ 575, 461

Gazeas, K. \& Stȩpień, K. 2008, astro-ph0803.0212

Kähler, H. 2004, A\&\&A 414, 317

Lucy, L. B. 1976, ApJ 205, 208

Stẹpień, K. 2004, in: A.K. Dupree \& A.O. Benz (eds.) Stars as Suns: Activity, Evolution and Planets, IAU Symp. No. 219 (San Francisco: Astr. Soc. of Pacific), p. 967

Stẹpień, K. 2006, AcA 56, 199

Wood, B. E., Müller, H. R., Zank, G. P., \& Linsky, J. L. 2002, ApJ 574, 412 